ESAIM: COCV

July 2006, Vol. 12, 398-408

DOI: $10.1051 /$ cocv:2006009
ESAIM: Control, Optimisation and Calculus of Variations

www.edpsciences.org/cocv

\title{
NEUMANN BOUNDARY VALUE PROBLEMS ACROSS RESONANCE*
}

\author{
Ginés López ${ }^{1}$ And JuAn-Aurelio Montero-SÁnchez ${ }^{1}$
}

\begin{abstract}
We obtain an existence-uniqueness result for a second order Neumann boundary value problem including cases where the nonlinearity possibly crosses several points of resonance. Optimal and Schauder fixed points methods are used to prove this kind of results.
\end{abstract}

Mathematics Subject Classification. 34B15, $47 \mathrm{H} 15$.

Received January 13, 2005. Revised May 11, 2005.

\section{INTRODUCTION}

The aim of this work is to give an existence and uniqueness result for elliptic boundary value problem of second order in resonance, that is, our hypotheses allow that the nonlinearity crosses several eigenvalues of the associated eigenvalue problem. Under suitable conditions, which include the regularity of the function $f$, the positivity of the function $\frac{\partial f}{\partial x}$ and a uniform upper bound for $\int_{0}^{\pi} \frac{\partial}{\partial x} f(t, x) \mathrm{d} t$, the problem

$$
\left.\begin{array}{c}
-x^{\prime \prime}(t)-\alpha(t) x^{\prime}(t)=f(t, x(t)), \text { in }[0, \pi] \\
A=x^{\prime}(0) ; B=x^{\prime}(\pi)
\end{array}\right\}
$$

has a unique solution (Th. 14), where $\alpha:[0, \pi] \rightarrow \mathbb{R}$ is a continuous function.

It is well known that in order to study the solutions of a boundary value problem for a second order operator, it is convenient to consider the interaction between the nonlinearity, $f(t, x)$, and the spectrum of the corresponding operator. See $[2,3,5]$.

Our motivation was a paper written by Huaizhong and Yong, [4], in which, for $\alpha \equiv 0$, the Theorem 14 below is obtained. For it, they use Pontryagin's maximum principle and the explicit expression of solutions for the associated linear problem to (1.1). When $\alpha \neq 0$ is a general continuous function, the explicit expression of solutions for the associated linear problem is unknown and so, the use of Pontryagin's maximum principle becomes difficult to handle, in order to obtain the main result (Th. 14), which is obtained in a different way.

In Section 2, we consider the linear problem associated with problem (1.1)

$$
\left.\begin{array}{c}
-x^{\prime \prime}-\alpha(t) x^{\prime}=\beta(t) x, t \in[0, \pi] \\
x^{\prime}(0)=x^{\prime}(\pi)=0
\end{array}\right\}
$$

where $\beta$ is a nonnegative, bounded and measurable function.

Keywords and phrases. Second order Newmann boundary condition, resonance, Pontryagin's maximum principle.

* Supported by D.G.E.S. Ministerio de Educación (Spain) BFM2002-02649.

1 Departamento de Análisis Matemático, Universidad de Granada, 18071 Granada, Spain; glopezp@ugr.es;jmontero@ugr.es

(C) EDP Sciences, SMAI 2006 
Now we define an optimal control problem for the above linear boundary value problem. We prove the existence of the optimal control and, after doing a qualitative study of the optimal solutions of (1.2), we obtain a lower bound for the minimum value of the functional cost. Moreover, we apply Pontryagin's maximum principle to describe the optimal control. Finally, in Section 3, applying the results of Section 2 and Schauder fixed-point theorem, we obtain our main result (Th. 14). Roughly speaking, we show that even though the function $f$ crosses some eigenvalues, an additional "energy" is necessary to get several solutions for the problem (1.1).

\section{LINEAR PROBLEM}

We consider the linear boundary problem

$$
\left.\begin{array}{c}
-x^{\prime \prime}-\alpha(t) x^{\prime}=\beta(t) x, t \in[0, \pi] \\
x^{\prime}(0)=x^{\prime}(\pi)=0
\end{array}\right\}
$$

where $\alpha$ is a continuous function on $[0, \pi]$ with $\beta \in L^{\infty}[0, \pi]$ and $\beta(t) \geq 0$ a.e. $t \in[0, \pi]$.

We start by studying the spectral structure of the associated eigenvalue problem.

$$
\left.\begin{array}{c}
-x^{\prime \prime}-\alpha(t) x^{\prime}=\lambda x, t \in[0, \pi] \\
x^{\prime}(0)=x^{\prime}(\pi)=0 .
\end{array}\right\}
$$

Lemma 1. The eigenvalues of the problem (2.3) are a sequence of real numbers $\lambda_{0}<\lambda_{1}<\cdots<\lambda_{n}<\cdots$, where $\lambda_{0}=0, \lim \lambda_{n}=+\infty$ and all of them are simple.

Proof. Multiplying equation (2.3) by e $\int_{0}^{t} \alpha(s) \mathrm{d} s$ we obtain the equivalent problem

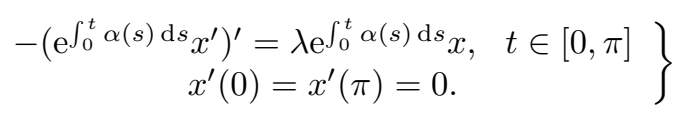

From [7] Theorem 27.II, we have that the eigenvalue problem (2.4), and so (2.3), has infinitely many simple real eigenvalues $\lambda_{0}<\lambda_{1}<\cdots<\lambda_{n}<\cdots$ and no other eigenvalues.

Since 0 is clearly an eigenvalue of (2.3), it remains only to see that $\lambda_{n}$ can't be negative for all $n \in \mathbb{N}$.

Assume that $\lambda_{n}<0$ for some $n$. Now, by integrating in (2.4) we obtain

$$
x^{\prime}(t)=-\mathrm{e}^{-\int_{0}^{t} \alpha(s) \mathrm{d} s} \int_{0}^{t} \mathrm{e}^{\int_{0}^{s} \alpha(\tau) \mathrm{d} \tau} \lambda_{n} x(s) \mathrm{d} s, \quad \forall t \in[0, \pi]
$$

where $x \in C^{1}[0, \pi]$ is the solution of $(2.3)$ with $\lambda=\lambda_{n}$ satisfying $x(0)=1$.

Since $\lambda_{n}<0$ and $x(0)>0$, one can deduce from $(2.5)$ that $x^{\prime}(t)>0, \forall t \in(0, \pi)$. Then $x(t)>0, \forall t \in[0, \pi]$. Doing $t=\pi$ in (2.5) one has that $\int_{0}^{\pi} \mathrm{e}^{\int_{0}^{s} \alpha(\tau) \mathrm{d} \tau} \lambda_{n} x(s) \mathrm{d} s=0$ which is a contradiction.

Choose a suitable admissible set $\Omega_{B}$ as follows:

$$
\Omega_{B}=\left\{\beta \in L^{\infty}[0, \pi] \backslash\{0\}: 0 \leq \beta(t) \leq B \text {, a.e. } t \in[0, \pi]\right. \text { and }
$$

(1.2) has nontrivial solution for $\beta=\beta(t)\}$,

where $\lambda_{1} \leq B$. Our control problem will be to find a function $\beta^{*} \in \Omega_{B}$ such that $\beta^{*}(t)$ minimizes the functional $J$ defined by

$$
J(\beta)=\int_{0}^{\pi} \beta(t) \mathrm{d} t, \forall \beta \in \Omega_{B},
$$


that is,

$$
J\left(\beta^{*}\right)=\min _{\Omega_{B}} J(\beta) .
$$

In order to prove the existence of a minimum in $\Omega_{B}$ for $J$ we note that every solution of (1.2), for $\beta \in \Omega_{B}$ has a zero.

Lemma 2. If $\beta \in \Omega_{B}$ and $x_{\beta}$ is a solution of (1.2) for that $\beta$, then $x_{\beta}$ has a zero.

Proof.

Arguing as in Lemma 1, we have

$$
x^{\prime}(\pi)=-\mathrm{e}^{-\int_{0}^{\pi} \alpha(s) \mathrm{d} s} \int_{0}^{\pi} \mathrm{e}^{\int_{0}^{s} \alpha(\tau) \mathrm{d} \tau} \beta(s) x(s) \mathrm{d} s=0,
$$

and we conclude the result.

Lemma 3. $\Omega_{B}$ is a weakly compact set of $L^{1}(0, \pi)$.

Proof. Since the identity map $i: L^{2}(0, \pi) \rightarrow L^{1}(0, \pi)$ is continuous for the weak topologies and $\Omega_{B}$ is bounded, it is enough to see that $\Omega_{B}$ is a weakly closed subset of $L^{2}(0, \pi)$.

Pick $\left\{\beta_{n}\right\} \subset \Omega_{B}$ a sequence and for each $n$, take $x_{n}$ a nontrivial solution of problem (1.2) for $\beta=\beta_{n}$. By linearity we can assume that

$$
\left\|x_{n}\right\|_{\infty}+\left\|x_{n}^{\prime}\right\|_{\infty}=1, \forall n \in \mathbb{N} .
$$

Consequently, the sequences $\left\{x_{n}\right\}$ and $\left\{x_{n}^{\prime}\right\}$ are uniformly bounded and so $\left\{x_{n}\right\}$ is a equicontinuous sequence. From the equation (1.2) for $x=x_{n}$ we deduce that $\left\{x_{n}^{\prime \prime}\right\}$ is a uniformly essentially bounded sequence. Hence, $\left\{x_{n}^{\prime}\right\}$ is also equicontinuous. By the Ascoli-Arzelà theorem, passing to a subsequence if necessary, we can assume that

$$
\left\{x_{n}\right\} \rightarrow x_{0}, \quad\left\{x_{n}^{\prime}\right\} \rightarrow y_{0} \quad(n \rightarrow \infty)
$$

uniformly on $[0, \pi]$ for suitable $x_{0}, y_{0}$ continuous functions on $[0, \pi]$. Now, it is clear that $y_{0}=x_{0}^{\prime}$ and $\left\|x_{0}\right\|_{\infty}+$ $\left\|x_{0}^{\prime}\right\|_{\infty}=1$, so $x_{0} \not \equiv 0$

From the boundness of the sequence $\left\{\beta_{n}\right\}$ in $L^{2}(0, \pi)$, we can assume, passing to a subsequence if necessary, that $\left\{\beta_{n}\right\}$ converges to $\beta_{0} \in L^{2}(0, \pi)$ for the weakly topology. Passing to the limit in the equation (1.2) for $x=x_{n}$ and $\beta=\beta_{n}$ we can deduce that $x_{0}$ is a solution of the problem (1.2) for $\beta=\beta_{0}$, taking into account that $\left\{x_{n}\right\}$ converges "strongly" to $x_{0}$ and $\left\{\beta_{n}\right\}$ goes weakly to $\beta_{0}$, both in $L^{2}(0, \pi)$.

In order to prove that $\beta_{0} \in \Omega_{B}$, it remains only to see that $\beta_{0} \not \equiv 0$.

By Lemma 2, for each $n$ there is $t_{n} \in(0, \pi)$ such that $x_{n}\left(t_{n}\right)=0$. We can assume that $t_{n} \rightarrow t^{*} \in[0, \pi]$. As $x_{n} \rightarrow x_{0}$ uniformly then $x_{0}\left(t^{*}\right)=0$. From the uniqueness for initial value problems for the equation (1.2), we deduce that $t^{*} \in(0, \pi)$, since $x_{0}^{\prime}(0)=x_{0}^{\prime}(\pi)=0$. Therefore $x_{0}$ is not a constant function and so $\beta_{0} \neq 0$ a.e. in $L^{2}(0, \pi)$. Finally, $\beta_{0} \in \Omega_{B}$.

Before proving the existence of minimum in $\Omega_{B}$ for $J$, we recall that a point $x$ of a set $C$ in a vector space is said to be an extreme point of $C$, if $x$ is not an interior point of any nontrivial segment contained in $C$. The set of all extreme points of $C$ will be denoted by $\operatorname{extr}(C)$.

Theorem 4. The functional $J$ attains its minimum at an admisible $\beta_{0} \in \Omega_{B}$ such that

$$
\beta_{0} \in \operatorname{extr}\left(\overline{c o}\left(\Omega_{B}\right)\right) \quad\left(\Rightarrow \beta_{0} \in \operatorname{extr}\left(\Omega_{B}\right)\right) .
$$

Moreover, $\min _{\Omega_{B}} J=\min _{\overline{c o}\left(\Omega_{B}\right)} J$. 
Proof. Observe that $\Omega_{B} \neq \emptyset$ because the eigenfunction associated to the eigenvalues $\lambda_{1}$ is a nontrivial solution of problem (1.2) for $\beta(t) \equiv \lambda_{1}$ and therefore $\beta(t) \equiv \lambda_{1}$ belongs to $\Omega_{B}$. Moreover, the closed convex hull of $\Omega_{B}, \overline{c o}\left(\Omega_{B}\right)$, is weakly compact in $L^{1}(0, \pi)$, applying Lemma 3 . We can see the functional $J$ defined on $\overline{c o}\left(\Omega_{B}\right)$, namely $\widetilde{J}$. Now, by Bauer's Theorem (see for instance [1, Th. V.1]), $\widetilde{J}$ attains its minimum on $\beta_{0} \in \operatorname{extr}\left(\overline{c o}\left(\Omega_{B}\right)\right)$. By [1, Prop. V.4], we obtain that in fact $\beta_{0} \in \operatorname{extr}\left(\Omega_{B}\right)$. Then $J\left(\beta_{0}\right)=\widetilde{J}\left(\beta_{0}\right)$ is the minimum value of $J$ on $\Omega_{B}$.

The following lemma exhibits some qualitative properties for the solutions of the problem (1.2) when $\beta$ is a point of minimum for $J$.

Lemma 5. Let $\beta_{1}$ be an element in $\Omega_{B}$. Assume that the problem (1.2) for $\beta=\beta_{1}$ has a nontrivial solution $x_{1}$ whose derivative has a zero in $(0, \pi)$. Then there is $\beta_{2}$ in $\Omega_{B}$ such that every nontrivial solution, $x_{2}$ of problem (1.2) for $\beta=\beta_{2}$ satisfies $x_{2}^{\prime}(t) \neq 0$ in $(0, \pi)$ and $\int_{0}^{\pi} \beta_{2}(t) \mathrm{d} t<\int_{0}^{\pi} \beta_{1}(t) \mathrm{d} t$.

Proof. Let $t_{1} \in(0, \pi)$ be a zero of $x_{1}^{\prime}$. By Lemma 2 there is $t_{2} \in(0, \pi)$ such that $x_{1}\left(t_{2}\right)=0$. The uniqueness of initial value problem for equation (1.2) gives $x_{1}^{\prime}\left(t_{2}\right) \neq 0$. It is clear that there is $0 \leq a<b \leq \pi$ such that $t_{2} \in(a, b), x_{1}^{\prime}(a)=x_{1}^{\prime}(b)=0$ and $x_{1}^{\prime}(t) \neq 0, \forall t \in(a, b)$. Clearly $0<b-a<\pi$.

Consider $F(t)=\int_{0}^{t} \frac{\mathrm{d} s}{1-K \mathrm{e}_{0}^{s} \alpha(\tau) \mathrm{d} \tau}+a, t \in(0, \pi)$ where $K<0$ is chosen such that the equality $F(\pi)=b$ holds (this is possible by virtue of the continuity of $F$ with respect to the parameter $K$ ). Note that $F$ defined above is the unique solution of

$$
\left.\begin{array}{c}
f^{\prime \prime}+\alpha(t) f^{\prime}=\alpha(t)\left(f^{\prime}\right)^{2} \\
f(0)=a, \quad f(\pi)=b
\end{array}\right\}
$$

Now, define $x_{2}(t)=x_{1}(F(t)), \forall t \in[0, \pi]$. One can check that $x_{2}$ is a solution of problem $(1.2)$ for $\beta=$ $F^{\prime}(t)^{2} \beta_{1}(F(t))$. Moreover, $x_{2}^{\prime}(t)=x_{1}^{\prime}(F(t)) F^{\prime}(t) \neq 0$, for $t \in(0, \pi)$. Taking $\beta_{2}=F^{\prime}(t)^{2} \beta_{1}(F(t))$ one has $\beta_{2} \in \Omega_{B}$, since

Consequently,

$$
F^{\prime}(t)=\frac{1}{1-K \mathrm{e}_{0}^{t} \alpha(\tau) \mathrm{d} \tau}<1, \forall t \in(0, \pi)
$$

$$
\int_{0}^{\pi} \beta_{2}=\int_{0}^{\pi} F^{\prime}(t)^{2} \beta_{1}(F(t)) \mathrm{d} t<\int_{0}^{\pi} F^{\prime}(t) \beta_{1}(F(t)) \mathrm{d} t=\int_{a}^{b} \beta_{1}(t) \mathrm{d} t \leq \int_{0}^{\pi} \beta_{1}(t) \mathrm{d} t .
$$

Corollary 6. If $\beta$ is a minimum for $J$, then every nontrivial solution $x(t)$ of the problem $(1.2)$ satisfies $x^{\prime}(t) \neq 0$ for all $t \in(0, \pi)$. In particular, $x$ has exactly one zero in $[0, \pi]$.

Note that the minimum of the functional $J$ is strictly positive, so we will estimate this minimum and for this we need to fix some notation.

In the subsequent lemmas we will be in the following setting

$$
\left\{\begin{array}{l}
\bullet \beta^{*} \text { is a minimum of } J \text { (Th. } 4 \text { ), } \\
\bullet x_{1}, x_{2} \text { are two solutions (so, linearly dependent) of the problem }(1.2) \text { with } \\
x_{1}(0)=1, x_{2}(\pi)=1 \text {, for } \beta=\beta^{*} \text {, and } \\
\bullet t^{*} \in(0, \pi) \text { is the unique point in }(0, \pi) \text { satisfying } x_{1}\left(t^{*}\right)=0, x_{2}\left(t^{*}\right)=0 \\
(\text { Cor. } 6) \text {. }
\end{array}\right.
$$

Lemma 7. $x_{1}(\pi) x_{2}(0)=1$.

Proof. It is clear that there is $c \in \mathbb{R} \backslash\{0\}$ such that $x_{1}=c x_{2}$. Hence, $x_{1}(\pi)=c x_{2}(\pi)=c$, and then $1=x_{1}(0)=c x_{2}(0)=x_{1}(\pi) x_{2}(0)$. 
Lemma $8 . \quad$ i)

$$
\int_{0}^{t^{*}} \beta^{*}(s) \mathrm{d} s>\frac{1-x_{1}(\pi)}{\pi} \mathrm{e}^{-2 \pi\|\alpha\|_{\infty}} .
$$

ii)

$$
\int_{t^{*}}^{\pi} \beta^{*}(s) \mathrm{d} s>\frac{1-x_{2}(0)}{\pi} \mathrm{e}^{-2 \pi\|\alpha\|_{\infty}}
$$

Proof.

i) Integrating in (2.5) for $x=x_{1}$ and $\lambda_{n}=\beta^{*}$ we deduce

$1-x_{1}(\pi)=\int_{0}^{\pi} \mathrm{e}^{-\int_{0}^{t} \alpha(\tau) \mathrm{d} \tau} \int_{0}^{t} \mathrm{e}^{\int_{0}^{s} \alpha(\tau) \mathrm{d} \tau} \beta^{*}(s) x_{1}(s) \mathrm{d} s \mathrm{~d} t$

$$
<\int_{0}^{\pi} \mathrm{e}^{t\|\alpha\|_{\infty}} \int_{0}^{t^{*}} \mathrm{e}^{s\|\alpha\|_{\infty}} \beta^{*}(s) \mathrm{d} s \mathrm{~d} t \leq \pi \mathrm{e}^{2 \pi\|\alpha\|_{\infty}} \int_{0}^{t^{*}} \beta^{*}(s) \mathrm{d} s .
$$

The second inequality is trivial. For the first inequality we used that the function $x_{1}$ is strictly positive in $\left[0, t^{*}\right)$, strictly negative in $\left(t^{*}, \pi\right]$ and $x_{1}(t) \leq 1$ in $[0, \pi]$ (by $(\mathbf{H})$ and Cor. 6).

ii) The proof is similar to the proof of i), by using that $x_{2}$ is strictly negative in $\left[0, t^{*}\right)$, strictly positive in $\left(t^{*}, \pi\right]$ and $x_{2}(t) \leq 1$ in $[0, \pi]$ (by $(\mathbf{H})$ and Cor. 6).

Now, we present the main tool of the section.

\section{Theorem 9.}

$$
\min _{\Omega_{B}} J>\frac{4}{\pi} \mathrm{e}^{-2 \pi\|\alpha\|_{\infty}} .
$$

Proof. From the previous lemma we obtain $J\left(\beta^{*}\right)>\frac{\mathrm{e}^{-2 \pi\|\alpha\|_{\infty}}}{\pi}\left(2-x_{1}(\pi)-x_{2}(0)\right)$. Put $a=-x_{1}(\pi), b=-x_{2}(0)$. From Corollary 6 and Lemma 7 it is clear that $a, b>0$ and $a b=1$, since $x_{1}(0)=1$ and $x_{2}(\pi)=1$. Then we conclude $a+b \geq 2$ and so the proof.

Remark. Observe that with the same computations as in Lemma 8, we obtain a better bound on the $\min _{\Omega_{B}} J$. Specifically,

$$
\min _{\Omega_{B}} J>\frac{4}{\left\|\mathrm{e}_{0}^{t} \alpha\right\|_{\infty} \int_{0}^{\pi} \mathrm{e}^{-\int_{0}^{t} \alpha} \mathrm{d} t} .
$$

However, we will use Theorem 9 for simplicity reasons.

Note that the estimation in Theorem 9 is independent of $B$, so as a consequence we obtain the following

Corollary 10. Let $\Omega=\left\{\beta \in L^{\infty}(0, \pi) \backslash\{0\}: \beta(t) \geq 0\right.$ a.e. $t \in[0, \pi]$ and the problem (1.2) has nontrivial solution $\}$. Then, $\int_{0}^{\pi} \beta(t) \mathrm{d} t>\frac{4}{\pi} \mathrm{e}^{-2 \pi\|\alpha\|_{\infty}}$ for each $\beta \in \Omega$.

The above corollary is used now to obtain uniqueness conditions on the problem (1.2), which generalize [4], Theorem 3. 
Corollary 11. Let $\beta \in L^{\infty}(0, \pi) \backslash\{0\}$ such that $\beta(t) \geq 0$ a.e. $t \in[0, \pi]$ and $\int_{0}^{\pi} \beta(t) d t \leq \frac{4}{\pi} e^{-2 \pi\|\alpha\|_{\infty}}$. Then for each $g \in L^{1}(0, \pi), R, S \in \mathbb{R}$ the boundary value problem

$$
\left.\begin{array}{c}
x^{\prime \prime}+\alpha(t) x^{\prime}+\beta(t) x=g(t), \quad t \in[0, \pi] \\
x^{\prime}(0)=R, x^{\prime}(\pi)=S,
\end{array}\right\}
$$

has a unique solution.

Proof. From Corollary 10 the problem (2.6) has at most one solution. Since the equation is linear, the uniqueness implies the existence.

In order to complete the study of our control problem, we describe now the functions $\beta \in \Omega_{B}$ where $J$ attains its minimum on $\Omega_{B}$ for $B>\lambda_{1}$.

Theorem 12. Assume $\alpha$ differentiable and let $B>\lambda_{1}$. Then, there is $\beta^{*} \in \Omega_{B}$ where $J$ attains its minimum on $\Omega_{B}$, with the following form:

$$
\beta^{*}(t)=\left\{\begin{array}{cc}
B, & 0 \leq t \leq t_{1} \\
0, & t_{1}<t<t_{2} \\
B, & t_{2} \leq t \leq \pi
\end{array}\right.
$$

with $t_{1}<t_{2} \in(0, \pi)$ satisfying the equation

$$
\begin{aligned}
0= & \exp \left\{-\int_{0}^{t_{2}} \alpha\right\} y\left(t_{2}\right) x^{\prime}\left(t_{1}\right) \\
& -\exp \left\{-\int_{0}^{t_{1}} \alpha\right\} x\left(t_{1}\right) y^{\prime}\left(t_{2}\right)-x^{\prime}\left(t_{1}\right) y^{\prime}\left(t_{2}\right) \int_{t_{1}}^{t_{2}} \exp \left\{-\int_{0}^{s} \alpha\right\} \mathrm{d} s
\end{aligned}
$$

where $\{x, y\}$ are the solutions of the equation (1.2) for $\beta=B$ satisfying $x(0)=y(\pi)=1$ and $x^{\prime}(0)=y^{\prime}(\pi)=0$.

Proof. Choose $B>\lambda_{1}$ and take $\beta^{*} \in \Omega_{B}$ such that $J$ attains its minimum on $\Omega_{B}$. Let $z_{0}$ be the solution of problem (1.2) for $\beta=\beta^{*}$ satisfying $z_{0}^{\prime}(0)=z_{0}^{\prime}(\pi)=0$ and $z_{0}(0)=1$. Setting $w_{0}=z_{0}^{\prime}$ we have that $\left(z_{0}, w_{0}\right)$ is a solution of the problem

$$
\left(\begin{array}{c}
z^{\prime} \\
w^{\prime}
\end{array}\right)=\left(\begin{array}{cc}
0 & 1 \\
-\beta^{*} & -\alpha
\end{array}\right)\left(\begin{array}{c}
z \\
w
\end{array}\right)
$$

with conditions $z(0)=1$ and $w(0)=0$. By Theorem 4 and Pontryagin's Maximum Principle (see for instance [6], Th. 4.1), we have $\exists(\lambda, \mu) \in \mathbb{R}^{2} \backslash\{(0,0)\}$, with $\lambda \geq 0$, and absolutely continuous functions $P, Q:[0, \pi] \rightarrow \mathbb{R}$ solutions of the following problem

$$
\left(\begin{array}{c}
P^{\prime} \\
Q^{\prime}
\end{array}\right)=-\left(\begin{array}{cc}
0 & -\beta^{*} \\
1 & -\alpha
\end{array}\right)\left(\begin{array}{c}
P \\
Q
\end{array}\right)
$$

with conditions $P(\pi)=0$ and $Q(\pi)=\mu$.

Furthermore,

Equivalently,

$$
\begin{gathered}
\left\langle\left(\begin{array}{c}
P \\
Q
\end{array}\right),\left(\begin{array}{cc}
0 & 1 \\
-\beta^{*} & -\alpha
\end{array}\right)\left(\begin{array}{c}
z_{0} \\
w_{0}
\end{array}\right)\right\rangle+\lambda \beta^{*}= \\
\min _{\phi \in[0, B]}\left\langle\left(\begin{array}{c}
P \\
Q
\end{array}\right),\left(\begin{array}{cc}
0 & 1 \\
-\phi & -\alpha
\end{array}\right)\left(\begin{array}{c}
z_{0} \\
w_{0}
\end{array}\right)\right\rangle+\lambda \phi \text { a.e. }[0, \pi] .
\end{gathered}
$$

$$
\left(\lambda-Q z_{0}\right) \beta^{*}=\min _{\phi \in[0, B]}\left(\lambda-Q z_{0}\right) \phi \text { a.e. }[0, \pi] .
$$

From (2.9) we deduce that

$$
\begin{gathered}
-Q^{\prime \prime}+(\alpha Q)^{\prime}=\beta^{*} Q \\
Q(\pi)=\mu, \quad Q^{\prime}(\pi)=\alpha(\pi) \mu .
\end{gathered}
$$


Observe that $q(t)=\mathrm{e}_{0}^{\int_{0}^{t} \alpha} z_{0}(t)$ satisfies $-q^{\prime \prime}+(\alpha q)^{\prime}=\beta^{*} q$ and $q(\pi)=\mathrm{e}^{\int_{0}^{\pi} \alpha} z_{0}(\pi), q^{\prime}(\pi)=\alpha(\pi) \mathrm{e}_{0}^{\int_{0}^{\pi} \alpha} z_{0}(\pi)$. Then, $Q(t)=\frac{\mu \mathrm{e}^{-\int_{0}^{\pi} \alpha}}{z_{0}(\pi)} \mathrm{e}^{\int_{0}^{t} \alpha} z_{0}(t)$.

If $\mu=0$ holds then $\lambda>0$ and $Q \equiv 0$. Now from (2.12) we obtain $\beta^{*} \equiv 0 \notin \Omega_{B}$. So, $\mu \neq 0$.

If $\lambda=0$ holds then from (2.12) we conclude that $\beta^{*} \equiv 0 \notin \Omega_{B}$ or $\beta^{*} \equiv B$ (not possible because $B>\lambda_{1}$ ), since the function $Q z_{0}$ does not change sing in $[0, \pi]$. Thus, $\lambda>0$.

Again from (2.12)

$$
\beta^{*}(t)=\left\{\begin{array}{r}
0, \text { if } \frac{\mu \mathrm{e}^{-\int_{0}^{\pi} \alpha}}{z_{0}(\pi)} \mathrm{e}_{0}^{t} \alpha z_{0}^{2}(t)<\lambda \\
B, \text { if } \frac{\mu \mathrm{e}^{-\int_{0}^{\pi} \alpha}}{z_{0}(\pi)} \mathrm{e}_{0}^{\int_{0}^{t} \alpha} z_{0}^{2}(t)>\lambda .
\end{array}\right.
$$

From Corollary $6, z_{0}$ is strictly monotone, then the number of elements in the set $\mathcal{A}=\{t \in[0, \pi]$ : $\left.\frac{\mu \mathrm{e}^{-\int_{0}^{\pi} \alpha}}{z_{0}(\pi)} \mathrm{e}_{0}^{t} \alpha z_{0}^{2}(t)=\lambda\right\}$ is either zero, one or two. The cases zero and one give $\beta^{*} \equiv 0$ in an interval $I$ containing 0 or $\pi$ and so, $z_{0}$ is constant on $I$, which is a contradiction with Corollary 6 . In the case that $\mathcal{A}$ has two elements, the same argument proves that the unique possibility for $\beta^{*}$ is

$$
\beta^{*}(t)=\left\{\begin{array}{cc}
B & \text { if } t \in\left(0, t_{1}\right) \\
0 & \text { if } t \in\left(t_{1}, t_{2}\right) \\
B & \text { if } t \in\left(t_{2}, \pi\right) .
\end{array}\right.
$$

Take $\{x, y\}$ the solutions of (1.2) with $\beta=B$ satisfying $x(0)=1, x^{\prime}(0)=0, y(\pi)=1$ and $y^{\prime}(\pi)=0$. Let $\beta^{*}$ be a function in $\Omega_{B}$ where $J$ attains its minimum value on $\Omega_{B}$. Then, there are some $t_{1}, t_{2} \in(0, \pi)$ such that

$$
\beta^{*}(t)=\left\{\begin{array}{cc}
B & \text { if } t \in\left(0, t_{1}\right) \\
0 & \text { if } t \in\left(t_{1}, t_{2}\right) \\
B & \text { if } t \in\left(t_{2}, \pi\right)
\end{array}\right.
$$

and hence, a nontrivial solution $x_{\beta^{*}}$ of (1.2) for $\beta=\beta^{*}$ can be written, when $B$ is not an eigenvalue of (2.3), in the way

$$
x_{\beta^{*}}(t)= \begin{cases}a x(t)+b y(t), & \text { if } t \in\left(0, t_{1}\right) \\ c+d \int_{0}^{t} \exp \left\{-\int_{0}^{s} \alpha\right\} \mathrm{d} s, & \text { if } t \in\left(t_{1}, t_{2}\right) \\ e x(t)+f y(t), & \text { if } t \in\left(t_{2}, \pi\right)\end{cases}
$$

for suitable $a, b, c, d, e, f \in \mathbb{R}$, since $\{x, y\}$ is a fundamental system of (1.2) with $\beta=\beta^{*}$. Now, we impose the continuity and differentiability on $x_{\beta^{*}}$ in $t_{1}$ and $t_{2}$ joint to $x_{\beta^{*}}^{\prime}(0)=x_{\beta^{*}}^{\prime}(\pi)=0$ and we obtain that the following system

$$
\begin{array}{r}
a x^{\prime}(0)+b y^{\prime}(0)=0 \\
a x\left(t_{1}\right)+b y\left(t_{1}\right)-c-d \int_{0}^{t_{1}} \exp \left\{-\int_{0}^{s} \alpha\right\} \mathrm{d} s=0 \\
a x^{\prime}\left(t_{1}\right)+b y^{\prime}\left(t_{1}\right)-d \exp \left\{-\int_{0}^{t_{1}} \alpha\right\}=0 \\
e x\left(t_{2}\right)+f y\left(t_{2}\right)-c-d \int_{0}^{t_{2}} \exp \left\{-\int_{0}^{s} \alpha\right\} \mathrm{d} s=0 \\
e x^{\prime}\left(t_{2}\right)+f y^{\prime}\left(t_{2}\right)-d \exp \left\{-\int_{0}^{t_{2}} \alpha\right\}=0 \\
e x^{\prime}(\pi)+f y^{\prime}(\pi)=0
\end{array}
$$


has a nontrivial solution $(a, b, c, d, e, f)$. Equivalently, the determinant $D$ of the following matrix must be 0:

$$
0=D=\operatorname{det}\left(\begin{array}{cccccc}
x^{\prime}(0) & y^{\prime}(0) & 0 & 0 & 0 & 0 \\
x\left(t_{1}\right) & y\left(t_{1}\right) & -1 & -\int_{0}^{t_{1}} \exp \left\{-\int_{0}^{s} \alpha\right\} \mathrm{d} s & 0 & 0 \\
x^{\prime}\left(t_{1}\right) & y^{\prime}\left(t_{1}\right) & 0 & -\exp \left\{-\int_{0}^{t_{1}} \alpha\right\} & 0 & 0 \\
0 & 0 & -1 & -\int_{0}^{t_{2}} \exp \left\{-\int_{0}^{s} \alpha\right\} \mathrm{d} s & x\left(t_{2}\right) & y\left(t_{2}\right) \\
0 & 0 & 0 & -\exp \left\{-\int_{0}^{t_{2}} \alpha\right\} & x^{\prime}\left(t_{2}\right) & y^{\prime}\left(t_{2}\right) \\
0 & 0 & 0 & 0 & x^{\prime}(\pi) & y^{\prime}(\pi)
\end{array}\right) .
$$

Computing the determinant $D$, one has

$$
\begin{aligned}
0=\exp \left\{-\int_{0}^{t_{2}} \alpha\right\} y\left(t_{2}\right) x^{\prime}\left(t_{1}\right)-\exp \left\{-\int_{0}^{t_{1}} \alpha\right\} x\left(t_{1}\right) y^{\prime}\left(t_{2}\right) \\
-\left(\int_{0}^{t_{2}} \exp \left\{-\int_{0}^{s} \alpha\right\} \mathrm{d} s-\int_{0}^{t_{1}} \exp \left\{-\int_{0}^{s} \alpha\right\} \mathrm{d} s\right) x^{\prime}\left(t_{1}\right) y^{\prime}\left(t_{2}\right)
\end{aligned}
$$

If $B$ is an eigenvalue of (2.3), then the above equation also holds, setting $x=y$.

Now, we finish this section by showing, as consequence, an improvement of Corollary 11, which generalizes [4], Theorem 2, giving a bound depending of $B$.

Corollary 13. Fix $B>\lambda_{1}$. Assume that $\alpha$ is differentiable and let $\beta \in L_{\infty}(0, \pi) \backslash\{0\}$ such that $0 \leq \beta(t) \leq B$ a.e. in $[0, \pi]$. Let $\{x, y\}$ solutions of equation (1.2) for $\beta=B$ satisfying $x(0)=y(\pi)=1$ and $x^{\prime}(0)=y^{\prime}(\pi)=0$. If $\int_{0}^{\pi} \beta(t) \mathrm{d} t \leq B\left(t_{1}+\pi-t_{2}\right)$, where $0<t_{1}<t_{2}<\pi$ is a solution of the algebraic equation

$$
\begin{aligned}
0=\exp \left\{-\int_{0}^{t_{2}} \alpha\right\} y\left(t_{2}\right) x^{\prime}\left(t_{1}\right)-\exp \left\{-\int_{0}^{t_{1}} \alpha\right\} x\left(t_{1}\right) y^{\prime}\left(t_{2}\right) \\
-\left(\int_{0}^{t_{2}} \exp \left\{-\int_{0}^{r} \alpha\right\} \mathrm{d} r-\int_{0}^{t_{1}} \exp \left\{-\int_{0}^{r} \alpha\right\} \mathrm{d} r\right) x^{\prime}\left(t_{1}\right) y^{\prime}\left(t_{2}\right)
\end{aligned}
$$

which minimizes the expression $t_{1}-t_{2}$, then for each $f \in L_{1}(0, \pi), C, D \in \mathbb{R}$ the boundary value problem

$$
\left.\begin{array}{c}
x^{\prime \prime}+\alpha(t) x^{\prime}+\beta(t) x=f(t), \quad t \in[0, \pi] \\
x^{\prime}(0)=C, x^{\prime}(\pi)=D,
\end{array}\right\}
$$

has a unique solution.

\section{Nonlinear PRoblem}

The results of the previous section will become essential to get the main theorem concerning the existence and uniqueness of solution for the following nonlinear boundary value problem.

$$
\left.\begin{array}{c}
-x^{\prime \prime}-\alpha(t) x^{\prime}=f(t, x), \quad t \in[0, \pi] \\
x^{\prime}(0)=A, x^{\prime}(\pi)=B,
\end{array}\right\}
$$


where $A, B \in \mathbb{R}, \alpha:[0, \pi] \rightarrow \mathbb{R}$ is a continuous function and the nonlinearity $f:[0, \pi] \times \mathbb{R} \rightarrow \mathbb{R}$ satisfies that $f, f_{x}$ are continuous on $[0, \pi] \times \mathbb{R}$.

Theorem 14. Assume that the following requirements are fulfilled:

i) $0 \leq f_{x}(t, x) \leq \beta(t)$ on $[0, \pi] \times \mathbb{R}$, where $\beta \in L^{\infty}(0, \pi)$ and satisfies $\int_{0}^{\pi} \beta(t) \mathrm{d} t \leq \frac{4 \exp \left\{-2 \pi\|\alpha\|_{\infty}\right\}}{\pi}$.

ii) For each $x \in C[0, \pi]$ one has $f_{x}(t, x(t)) \neq 0 \quad$ a.e. on ${ }^{\pi}[0, \pi]$ and $\int_{0}^{\pi} \exp \left\{\int_{0}^{s} \alpha(\tau) \mathrm{d} \tau\right\} f(s, 0) \mathrm{d} s=0$.

Then, the problem (1.1) has a unique solution.

Proof. We first prove the uniqueness. Without loss of generality we can assume that $A=B=0$. Pick $x_{1}, x_{2}$ solutions of the problem (1.1), then $x(t)=x_{1}(t)-x_{2}(t)$ is a solution of the problem

$$
\left.\begin{array}{c}
-x^{\prime \prime}-\alpha(t) x^{\prime}=x \int_{0}^{1} f_{x}\left(t, x_{2}+\theta x\right) \mathrm{d} \theta, t \in[0, \pi] \\
x^{\prime}(0)=x^{\prime}(\pi)=0
\end{array}\right\} .
$$

Take $\beta_{0}(t)=\int_{0}^{1} f_{x}\left(t, x_{2}+\theta x\right) \mathrm{d} \theta$. Now, $x$ is a solution of the problem (1.2) for $\beta=\beta_{0}$. From our requirements $\beta_{0} \in L^{\infty}(0, \pi), 0 \leq \beta_{0}(t)$ a.e. on $[0, \pi]$ and $\int_{0}^{\pi} \beta_{0} \leq \frac{4 \exp \left\{-2 \pi\|\alpha\|_{\infty}\right\}}{\pi}$. Then, applying Corollary $10, x \equiv 0$.

The existence of solution will be obtained by an argument of Schauder fix point type. To do it, we write the nonlinear problem (1.1) in the equivalent form

$$
\left.\begin{array}{c}
-x^{\prime \prime}-\alpha(t) x^{\prime}=b(t, x) x+f(t, 0), t \in[0, \pi] \\
x^{\prime}(0)=x^{\prime}(\pi)=0
\end{array}\right\}
$$

where $b(t, x)=\int_{0}^{1} f_{x}(t, \theta x) \mathrm{d} \theta$. Let $X=\left\{x \in C^{1}[0, \pi]: x^{\prime}(0)=x^{\prime}(\pi)=0\right\}$ provided with the norm $\|x\|_{X}=$ $\sup _{[0, \pi]}|x(t)|+\sup _{[0, \pi]}\left|x^{\prime}(t)\right|$. It is known that $\left(X,\|\cdot\|_{X}\right)$ is a Banach space. Define the operator $T: X \rightarrow X$ as $x \rightarrow T x=y_{x}$, where $y_{x}$ is the solution of the linear problem

$$
\left.\begin{array}{c}
-y^{\prime \prime}-\alpha(t) y^{\prime}=b(t, x) y+f(t, 0), \quad t \in[0, \pi] \\
y^{\prime}(0)=y^{\prime}(\pi)=0 .
\end{array}\right\}
$$

Observe that by Corollary 11, the above problem has unique solution and then the operator $T$ is well defined. Let us see that $T$ is bounded, i.e., $\exists M>0$ such that $\|T x\|_{X} \leq M$ for each $x \in X$. Otherwise, there exists a sequence $\left\{x_{n}\right\} \in X$ satisfying $\left\|y_{x_{n}}\right\|_{X} \rightarrow+\infty$. From the estimate

$$
0 \leq b\left(t, x_{n}\right) \leq \beta(t), \quad t \in[0, \pi],
$$

we deduce the existence of a sequence, noted again by $\left\{b\left(t, x_{n}\right)\right\}$, such that $\left\{b\left(t, x_{n}\right)\right\} \rightarrow \beta_{1}$, where the weak limit function satisfies

$$
0 \leq \beta_{1}(t) \leq \beta(t), t \in[0, \pi] .
$$

We know that $y_{n}:=y_{x_{n}}$ is the solution defined by problem (3.22) and then

$$
\int_{0}^{\pi} y_{n}^{\prime} h^{\prime}-\int_{0}^{\pi} \alpha y_{n}^{\prime} h=\int_{0}^{\pi} b\left(t, x_{n}\right) y_{n} h+\int_{0}^{\pi} f(t, 0) h, \forall h \in H^{1}(0, \pi), \forall n \in \mathbb{N} .
$$

By normalizing $z_{n}(t):=\frac{y_{n}(t)}{\left\|y_{n}\right\|_{X}}$, we obtain

$$
\int_{0}^{\pi} z_{n}^{\prime} h^{\prime}-\int_{0}^{\pi} \alpha z_{n}^{\prime} h=\int_{0}^{\pi} b\left(t, x_{n}\right) z_{n} h+\frac{1}{\left\|y_{n}\right\|_{X}} \int_{0}^{\pi} f(t, 0) h, \forall h \in H^{1}(0, \pi), \forall n \in \mathbb{N} .
$$


From the previous equation one deduces that $z_{n}^{\prime \prime}$ are uniformly bounded, then by an Ascoli-Arzela argument $\left\{z_{n}\right\} \rightarrow z$ and $\left\{z_{n}^{\prime}\right\} \rightarrow z^{\prime}$ uniformly on $[0, \pi]$. Taking limit in the corresponding expressions, $z \neq 0$ (recall that $\left.\|z\|_{X}=1\right)$ is a weak solution of

$$
\int_{0}^{\pi} z^{\prime} h^{\prime}-\int_{0}^{\pi} \alpha z^{\prime} h=\int_{0}^{\pi} \beta_{1} z h, \forall h \in H^{1}(0, \pi) .
$$

Now, if one takes $h(t)=\mathrm{e}^{\int_{0}^{t} \alpha(\theta) \mathrm{d} \theta}$ in $(3.23)$, one obtains

$$
0=\int_{0}^{\pi} \mathrm{e}^{\int_{0}^{s} \alpha(\theta) \mathrm{d} \theta}\left[b\left(s, x_{n}\right) y_{n}+f(s, 0)\right] \mathrm{d} s,
$$

and hence, by hypothesis ii)

$$
\int_{0}^{\pi} \mathrm{e}^{\int_{0}^{s} \alpha(\theta) \mathrm{d} \theta} b\left(s, x_{n}\right) y_{n}=0 .
$$

This implies that the function $y_{n}$ has a zero in $[0, \pi]$ and consequently also $z$. As $z$ is nontrivial, the zero is actually in $(0, \pi)$ and so, $z$ is a nonconstant function. Thus, it follows from $(3.24)$ that $\beta_{1}$ is in $L^{\infty}(0, \pi) \backslash\{0\}$. On the other hand, hypothesis $i$ ) of theorem and Corollary 11 imply that $z \equiv 0$ which is a contradiction. Therefore, the operator $T$ is bounded.

Let us see now that the operator $T: X \rightarrow X$ is continuous. Take a convergent sequence $\left\{x_{n}\right\} \rightarrow x_{0} \in X$. Then, we need to show that $y_{n} \rightarrow y_{0}$ in $X$, where $y_{n}$ and $y_{0}$ are the corresponding solutions of problem (3.22) for $x=x_{n}$ and $x=x_{0}$, respectively. If the sequence is not convergent, then $\exists \eta>0$ and a subsequence $y_{n^{\prime}} \notin B_{X}\left(y_{0}, \eta\right), \forall n \in \mathbb{N}$. On the other hand, we know that $y_{n^{\prime}}^{\prime \prime}$ is uniformly bounded in $X$ (taking into account the equation (3.22) and the boundness of operator $T$ ), thus there exists a new subsequence $y_{n^{\prime \prime}} \rightarrow y$, which converges to some $y \in X$. Passing to the limit in the equation satisfied by $y_{n^{\prime \prime}}$, the uniqueness of solution for problem (3.22) implies that $y \equiv y_{0}$ which is a contradiction. Therefore, $y_{n} \rightarrow y_{0}$ and then operator $T$ is continuous.

To finish the proof, consider $T: B_{X}(0, M) \subset X \rightarrow X$ and arguing as above it is possible to show that $T$ is a compact operator. Then, Schauder's fix point theorem does the rest.

Remark. Note that the second condition in i) of Theorem 14 is only a $L^{1}$-norm condition for the function $\beta$, and so, a such $\beta$ can take any positive value. For example, fix $\gamma>0$ and take $\beta=\gamma \chi_{I}$, where $I$ is a subinterval of $[0, \pi]$ with length small enough, and $\chi_{I}$ is the characteristic function of $I$. Therefore, the Theorem 14 include cases where there is interaction between the nonlinearity $f_{x}$ and any positive eigenvalue of the spectrum. This interaction also exists from the right in zero. This phenomena is known in the literature as resonant phenomena.

To finish, we show a nonlinear version of Corollary 13 which generalizes [4], Theorem A.

Corollary 15. Fix $B>\lambda_{1}$ and suppose $\alpha$ differentiable. Assume that the following requirements are fulfilled:

i) $0 \leq f_{x}(t, x) \leq \beta(t) \leq B$ on $[0, \pi] \times \mathbb{R}$, where $\beta \in L^{\infty}(0, \pi)$ and satisfies $\int_{0}^{\pi} \beta(t) \mathrm{d} t \leq B\left(t_{1}+\pi-t_{2}\right)$. Here $0<t_{1}<t_{2}<\pi$ are defined as in Corollary 13 .

ii) For each $x \in C[0, \pi]$ one has $f_{x}(t, x(t)) \neq 0 \quad 0 \quad$ a.e. $\quad$ on $[0, \pi]$ and $\int_{0}^{\pi} \exp \left\{\int_{0}^{s} \alpha(\tau) \mathrm{d} \tau\right\} f(s, 0) \mathrm{d} s=0$.

Then, the problem (1.1) has a unique solution.

Acknowledgements. We want thank to Professor A. Cañada for proposing the problem to us and for his fruitful comments. 


\section{REFERENCES}

[1] B. Beauzamy, Introduction to Banach Spaces and their Geometry. North Holland, New York. Mathematics Studies 68 (1982).

[2] P. Hartman and A. Wintner, On an oscillation criterion of Liapunoff. Amer. J. Math. 73 (1951) 885-890.

[3] A.C. Lazer and D.E. Leach, On a nonlinear two-point boundary value problem. J. Math. Anal. Appl. 26 (1969) $20-27$.

[4] Y. Li and H. Wang, Neumann boundary value problems for second order ordinary differential equations across resonance. SIAM J. Control Optim. 33 (1995) 1312-11325.

[5] J. Mawhin, J.R. Ward and M. Willem, Variational methods and semi-linear elliptic equations. Arch. Rational Mech. Anal. 95 (1986) 269-277.

[6] E.R. Pinch, Optimal Control and the Calculus of Variations. Oxford University Press, New York (1993).

[7] W. Walter, Ordinary differential equations. Springer-Verlag, New York, Graduate Texts in Math. 182 (1998). 